\title{
Gut microbiome: a key player in cancer immunotherapy
}

\author{
Yasuyuki Shigematsu, Kentaro Inamura \\ Division of Pathology, The Cancer Institute, Department of Pathology, The Cancer Institute Hospital, Japanese Foundation for Cancer Research, \\ Tokyo, Japan \\ Correspondence to: Kentaro Inamura, MD, PhD. Division of Pathology, The Cancer Institute, Japanese Foundation for Cancer Research, 3-8-31 \\ Ariake, Koto-ku, Tokyo 135-8550, Japan. Email: kentaro.inamura@jfcr.or.jp. \\ Comment on: Routy B, Le Chatelier E, Derosa L, et al. Gut microbiome influences efficacy of PD-1-based immunotherapy against epithelial tumors. \\ Science 2018;359:91-7.
}

Submitted Oct 04, 2018. Accepted for publication Oct 10, 2018.

doi: 10.21037/hbsn.2018.10.02

View this article at: http://dx.doi.org/10.21037/hbsn.2018.10.02

Immune checkpoint inhibitors (ICIs) have revolutionized the therapeutic approaches used to treat cancer. Immune checkpoint molecules located on tumor cells interact with receptors on cytotoxic CD8+ $\mathrm{T}$ cells (a type of effector $\mathrm{T}$ lymphocyte) and consequently lead to evasion of tumor cells from the immune system. ICIs stimulate $\mathrm{T}$ cellmediated antitumor immunity by inhibiting the interactions between immune checkpoint molecules and inhibitory $\mathrm{T}$ cell receptors. The most widely used ICI is a monoclonal antibody that targets the interaction between PD-1 and its ligand PD-L1. PD-1 blockade, used as an immunotherapy, has been shown to be effective against a wide range of metastatic malignancies. However, patient responses are heterogeneous and often transient; furthermore, a high proportion of cancer patients are resistant to this immunotherapy (1-3). As a result, a substantial focus on biomarker discovery is underway to accurately predict clinical responses to PD-1 blockade. Biomarkers such as tumor mutational burden and immune cell infiltrates have been associated with responses to immunotherapy $(4,5)$; however, such tumor- and immune-based biomarkers lack robust sensitivity and specificity.

Accumulating evidence has demonstrated that abnormal microbiota-host interactions are associated with various malignancies, including gastric, colorectal, gallbladder, and liver cancers $(6,7)$. Recent studies have also indicated that microbiota-host interactions may affect responsiveness to immunotherapies by modulating innate and adaptive immunity $(8,9)$. Because the gut microbiota has a pronounced modulatory effect on the immune system, it may enhance ICI sensitivity. These findings, however, were primarily derived from studies performed in murine models, and little information is available about the functional impact of the human gut microbiota on therapeutic outcomes for cancer patients.

A recent study by Routy et al. demonstrated that the diversity and composition of the gut microbiota play critical roles in the response to PD-1 blockade and may have a prognostic value in cancer (10). The authors examined interactions between the gut microbiota and response to $\mathrm{PD}-1$ blockade in patients with non-smallcell lung carcinoma, renal cell carcinoma, and urothelial carcinoma. They observed that antibiotic-related dysbiosis was associated with a lower response to PD-1 blockade and a shorter survival period compared to patients who did not use antibiotics, suggesting that disruption of microbial networks and loss of specific bacterial species can affect the efficacy of immunotherapy. A comparison between the fecal microbiota of responders to PD-1 blockade and that of non-responders identified increased abundance of Akkermansia muciniphila (A. muciniphila) in patients who experienced longer survival. These authors confirmed the importance of the gut microbiota in immunotherapy by demonstrating that oral supplementation with $A$. muciniphila or fecal microbiota transplantation (FMT) from responders restored the efficacy of PD-1 blockade in murine models. These results indicate that the gut microbiota is not only a predictor but also a regulator of the response to PD-1 blockade. Routy et al. showed that tumors from mice that received FMT from responders contained a high density of CCR9+CXCR3+CD4+ T cells (10). These authors found that $A$. muciniphila induces dendritic cells to secrete IL- 
12, which is a critical component of the anticancer effect of PD-1 blockade. The recruitment of CCR9+CXCR3+CD4+ $\mathrm{T}$ cells into mouse tumor beds enhanced the effect of PD-1 blockade in an IL-12-dependent manner. These findings suggest that cytokines induced by microbiotahost interactions increase the efficacy of PD-1 blockade by modulating the immunosuppressive tumor microenvironment.

The association between the gut microbiota and immunotherapy outcomes remains unclear. A subset of patients with a favorable gut microbiota did not benefit from PD-1 blockade. Indeed, Routy et al. detected the presence of $A$. muciniphila in the feces of one-third of patients who progressed or died without any response (10). This fact suggests that the gut microbiota is not a perfect biomarker for the prediction of sensitivity to PD-1 blockade. Host responses to the gut microbiota may play critical roles in eliciting the beneficial effects of immunotherapy. Distinct bacterial species have also been proposed to contribute to sensitivity to PD-1 blockade in melanoma patients $(11,12)$; therefore, no universal bacterial species may determine the sensitivity to PD-1 blockade. These variations may be associated with the histological type of the tumors examined, environmental factors, study populations, or analytical methods. The complex interactions between the microbiota and tumor-immune microenvironment must be studied in detail to comprehend whether a universal or individual gut microbiota fingerprint is associated with immunotherapeutic sensitivity across various histological tumor types.

The discovery of bacterial species that enhance antitumor immunity as well as reduce immune-related adverse events (e.g., colitis and pneumonitis) is an important goal in clinical practice. The identification of specific causative bacteria may allow modulation of cancer progression, raising the possibility of precision immunotherapies. Targeting the microbiota as a novel immunotherapy biomarker will help the frontiers of precision medicine in providing improved patient care.

\section{Acknowledgements}

None.

\section{Footnote}

Conflicts of Interest: The authors have no conflicts of interest to declare.

\section{References}

1. Borghaei H, Paz-Ares L, Horn L, et al. Nivolumab versus Docetaxel in Advanced Nonsquamous Non-Small-Cell Lung Cancer. N Engl J Med 2015;373:1627-39.

2. Motzer RJ, Escudier B, McDermott DF, et al. Nivolumab versus Everolimus in Advanced Renal-Cell Carcinoma. N Engl J Med 2015;373:1803-13.

3. Topalian SL, Hodi FS, Brahmer JR, et al. Safety, activity, and immune correlates of anti-PD-1 antibody in cancer. $\mathrm{N}$ Engl J Med 2012;366:2443-54.

4. Rizvi NA, Hellmann MD, Snyder A, et al. Cancer immunology. Mutational landscape determines sensitivity to PD-1 blockade in non-small cell lung cancer. Science 2015;348:124-8.

5. Spranger S, Bao R, Gajewski TF. Melanoma-intrinsic betacatenin signalling prevents anti-tumour immunity. Nature 2015;523:231-5.

6. Schwabe RF, Jobin C. The microbiome and cancer. Nat Rev Cancer 2013;13:800-12.

7. Yoshimoto S, Loo TM, Atarashi K, et al. Obesity-induced gut microbial metabolite promotes liver cancer through senescence secretome. Nature 2013;499:97-101.

8. Sivan A, Corrales L, Hubert N, et al. Commensal Bifidobacterium promotes antitumor immunity and facilitates anti-PD-L1 efficacy. Science 2015;350:1084-9.

9. Vetizou M, Pitt JM, Daillere R, et al. Anticancer immunotherapy by CTLA-4 blockade relies on the gut microbiota. Science 2015;350:1079-84.

10. Routy B, Le Chatelier E, Derosa L, et al. Gut microbiome influences efficacy of PD-1-based immunotherapy against epithelial tumors. Science 2018;359:91-7.

11. Gopalakrishnan V, Spencer CN, Nezi L, et al. Gut microbiome modulates response to anti-PD-1 immunotherapy in melanoma patients. Science 2018;359:97-103.

12. Matson V, Fessler J, Bao R, et al. The commensal microbiome is associated with anti-PD-1 efficacy in metastatic melanoma patients. Science 2018;359:104-8.

Cite this article as: Shigematsu Y, Inamura K. Gut microbiome: a key player in cancer immunotherapy. HepatoBiliary Surg Nutr 2018;7(6):479-480. doi: 10.21037/ hbsn.2018.10.02 